\title{
Comparison of the Roles of Lymphatic Vessels and Blood Vessels in the Recurrence of Pterygia: A Retrospective Study
}

\author{
Wei Zhao1, Tao Wang², Jiezheng Yang2, Shiyi Yin², Ting Wang2, Lei Zhong2, Lili Chen1*, \\ Shiqi Ling ${ }^{2 *}$ \\ ${ }^{1}$ Department of Ophthalmology, Guangzhou Women and Children's Medical Center, Guangzhou Medical University, \\ Guangzhou, China \\ ${ }^{2}$ Department of Ophthalmology, The Third Affiliated Hospital, Sun Yat-sen University, Guangzhou, China \\ Email: *dorisgaosiyu@163.com, *lingshiqi123@163.com
}

How to cite this paper: Zhao, W., Wang, T., Yang, J.Z., Yin, S.Y., Wang, T., Zhong, L., Chen, L.L. and Ling, S.Q. (2020) Comparison of the Roles of Lymphatic Vessels and Blood Vessels in the Recurrence of Pterygia: A Retrospective Study. Open Journal of Ophthalmology, 10, 142-153. https://doi.org/10.4236/ojoph.2020.102016

Received: March 31, 2020

Accepted: May 12, 2020

Published: May 15, 2020

Copyright $\odot 2020$ by author(s) and Scientific Research Publishing Inc. This work is licensed under the Creative Commons Attribution International License (CC BY 4.0).

http://creativecommons.org/licenses/by/4.0/

(c) (i) Open Access

\begin{abstract}
Purpose: Increased conjunctival arteries and lymphangions accelerate the cellular immune response in recurrent pterygium, however, which plays a more important role warrants further investigation. The aim of the study is to compare the roles of lymphatic and blood vessels in pterygium recurrence. Methods: Histological sections from 48 excised recurrent pterygia (including 14 Grade 1, 20 Grade 2, and 14 Grade 3 tissues) were examined. Histological sections from seven nasal epibulbar conjunctival segments served as normal controls. Blood and lymphatic vessels were evaluated and compared according to blood microvessel density (BMD), blood vascular area (BVA), lymphatic microvessel density (LMD), and lymph-vascular area (LVA). Furthermore, the following relationships were analyzed: LMD and pterygium recurrence time (RT), LVA and RT, BMD and RT, BVA and RT. Results: Compared to LVA, LMD, BVA, and BVD values in normal control tissues, these values were markedly enhanced in recurrent pterygia tissues. The LMD/BMD and LVA/BVA ratios were significant increased in Grade 2 and 3 recurrent pterygia, suggesting that development of conjunctival lymphangions was not proportional to blood vessel growth. LMD, LVA and BVA were significantly correlated with RT for all grades of pterygia, while BMD was the only factor correlated with RT in Grade 1 pterygia. Moreover, no significant correlation was found between BMD and RT in Grade 2 and 3 pterygia. Conclusion: Compared to blood vessels, lymphangions might have a greater impact on pterygium recurrence.
\end{abstract}




\section{Keywords}

Relationship, Hemangiogenesis, Lymphangiogenesis, Pterygia, Recurrence

\section{Introduction}

A pterygium is a neoplasm that exhibits wing-shaped, fibrous vascular histology from the bulbi conjunctiva to the cornea; this pathology may cause blurring of vision, lacrimation, irritation, and foreign body sensation [1]. Although pterygia are benign, their recurrent nature is troublesome. Various adjunctive treatments, including radiotherapy, chemotherapy, and surgical transplants, have been employed to prevent recurrence. However, according to many studies, the recurrence rates of pterygia, ranging between $7.5 \%$ and $44.4 \%$, remain high and undermine the present operative methods and adjuvant treatments [2] [3]. Therefore, studies on the recurrence of pterygia have received increased attention in recent years.

Immunologic mechanisms, including $\mathrm{CD}_{4}^{+} \mathrm{T}$ cell-mediated immunity, contribute to the development and recurrence of pterygia [4] [5] [6]. However, at least two steps are required to complete this cellular immune response. First, antigenic material, such as antigen presenting cells exiting from the pterygium to cervical lymph glands, is indispensable in accelerating alloimmunization. Second, immune effector cells and factors generated in lymph nodes, such as T-lymphocytes, mast cells and plasma cells, enter the ocular surface to induce immune injuries. In our recent studies, both conjunctival hemangiogenesis and lymphangiogenesis occurred and were closely related in recurrent pterygia [7] [8]. Arteries provide a pathway of entry for immune cells, representing the centrifugal nerve arm of an immune reflex arc, while conjunctival lymphangiogenesis represents the esodic nerve arm of an immunity reflex pathway, which offers a path from the ocular surface to local lymph glands [9] [10] [11]. Increased conjunctival arteries and lymphangions accelerate the cellular immune response and provide new insights into recurrent pterygium treatment. However, the question of whether blood vessels or lymphatic vessels are more important in the recurrence of pterygia warrants further investigation. This investigation is needed because a significant relationship exists between hemangiogenesis and lymphangiogenesis. Additionally, the outgrowth of blood vessels is considered a common factor in puerperal events that occur with the outgrowth of lymphangions [11] [12] [13] [14]. However, to our knowledge, no studies are available on this topic.

The purpose of the current research is to compare the effects of hemangiogenesis and lymphangiogenesis on pterygium recurrence. Survey results from this research may extend our understanding of the mechanisms of pterygium development and recurrence. 


\section{Subjects and Methods}

\subsection{Ethical Approval}

This research was conducted according to the tenets of the Declaration of Helsinki for research involving human subjects and was approved by the IRB of the 3rd Affiliated Hospital of Sun Yat-sen University (NO: [2018] 02-009-01).

\subsection{Patients}

A total of 48 patients with recurrent pterygia (twenty males and twenty-eight females) with an average age of 61.9 years (range: 35 to 70 years) were enrolled in the study at the Department of Ophthalmology of the 3rd Affiliated Hospital of Sun Yat-sen University from January 2018 to June 2019. Clinical assessments for pterygia were conducted as previously described by the same ophthalmologist [4]. Briefly, based on objective indications (vascular distribution, conjunctival hyperemia and edema, relevant thickness of fiber vascular lesions, and common eye redness), a preoperative score of 1-3 was assigned (1+, mild; $2+$, moderate; $3+$, severe). All pterygium patients underwent surgical excision of the pterygium immediately after meeting the diagnostic criteria for recurrent pterygia, as defined by fibrovascular regrowth past the limbus in a previously compromised area. All of the lesions were located on the nasal side and only the heads of the pterygia were included as pterygium samples. The size of the pterygium, including the horizontal extension onto the cornea from the limbus and the width of the base at the limbus, was measured (in millimeters) with a slitlamp using a slit beam of light. Seven nasal epithelium conjunctival sections near the limbus that were resected during cataract operations served as normal controls.

\subsection{The Calculation of Pterygium Recurrence Time (RT)}

Before the second pterygium excision, each patient was asked about their medical history, and the timepoint at which the initial pterygium surgery was performed was recorded according to the patient's memory. The RT was calculated as the duration between the time of primary resection and the time of recurrent pterygium resection.

\subsection{Immunohistochemistry}

After fixation for 24 hours in 10\% neutral formaldehyde solution, samples were embedded in petroleum wax, serially sectioned into $4-\mu \mathrm{m}$-thick sections $(20$ segments per specimen), rehydrated with an ethanol-water gradient, and cleaned with distilled water. Endogenous peroxidase activity was blocked by incubation with $30 \mathrm{ml} / \mathrm{L}$ catalase for 20 minutes. Next, histological sections were subjected to high-pressure steam treatment at $121^{\circ} \mathrm{C}$ in $10 \mathrm{mmol} / \mathrm{L}$ citrate buffer ( $\mathrm{pH} 6.0$ ) for 10 minutes for antigen retrieval and cooled at room temperature for $30 \mathrm{mi}-$ nutes. Then, sections were incubated with the following primary antibodies for 3 hours: mouse anti-human lymphatic vessel endothelial hyaluronan receptor 1 
(LYVE-1) monoclonal antibody (R \& D systems, MN, USA) and mouse anti-human cluster of differentiation 31 (CD31) ( $\&$ D systems, MN, USA). Biotin vitamin-labeled rabbit anti-mouse immunoglobulin was used as the secondary antibody. SABC-peroxidase was used for immune detection. The slides were labeled with diaminobenzidine and counterstained with hematoxylin to detect peroxidase activity.

\subsection{Lymphatic Microvessel Density (LMD) and Blood Microvessel Density (BMD)}

LMD and BVD were assessed independently by two researchers without previous knowledge of the experimental details, and were duplicated once. $\mathrm{CD}_{31}{ }^{(+)} \mathrm{LYVE}-1^{(-)}$ vessels of excised pterygium sections were identified as blood vessels, and $\mathrm{CD}_{31}{ }^{(+)}$LYVE-1 ${ }^{(+)}$vessels were identified as lymphangions. Each sample was separated into 20 sections. Each section was analyzed by light microscopy (Nikon, Eclipse 200). Under $100 \times$ magnification $\left(0.78 \mathrm{~mm}^{2}\right)$, the five most highly lymph-vascularized regions were identified, and the number of immunostained lymphangions was quantified. Only vessels displaying representative morphology (lumen) were considered microangiolymphs. The LMD of each sample is represented by the average value (number of vessels in 100 microscopic fields/100). Similarly, the total number of blood vessels in 100 fields was quantified and divided by 100 to obtain the BMD.

\subsection{Quantification of Blood Vascular Area (BVA) and Lymph-Vascular Area (LVA)}

Sections labeled with $\mathrm{CD}_{31}$ or LYVE-1 were observed with a Zeiss Axioskop microscope, and images were projected onto a Sony PVM1440QM video monitor with a Sony CCDIRIS camcorder. Digitized images were captured with a Fujix $\mathrm{HC}-1000$ 3CCD HR color camcorder. $\mathrm{CD}_{31}{ }^{(+)}$vessels included blood vessels and lymphatic vessels, while LYVE- $1^{(+)}$vessels only included lymphatic vessels. After a preparatory scan of each portion at low power, five high-vessel density regions were identified and imaged at high power $(100 \times)$. Conversion of computer images to a thresholded primitive binary format was performed to examine the lumens of the stained tissue while minimizing background histological staining. These images were analyzed using an internal image analysis program that showed the proportions of the image occupied by immunostained blood vessels and lymphangions (Axiovision 4.7.2; Carl Zeiss, Jena, Germany).

\subsection{Statistical Analysis}

Analysis of the differences between the 2 groups was conducted using a paired Student's t-test (SPSS 12.0 statistical software, SPSS Incorporation, Chicago, Illinois, United States of America). Pearson's correlation analysis was applied to analyze the relationships between RT and LMD, RT and LVA, RT and $\mathrm{BMD}$, and RT and BVA. Values are presented as the average \pm SD. All reported $\mathrm{P}$-values are two-tailed, and statistical significance was defined at the $\alpha=0.05$ 
level.

\section{Results}

\subsection{Composition of the Pterygia Study Group}

Of the 48 patients, a Grade 1 pterygium was found in 14 (29\%); Grade 2, in 20 (42\%); and Grade 3, in 14 (29\%). Among recurrent pterygia patients, RT in Grade 1 pterygium was $(25.14 \pm 4.49)$ months, $(19.92 \pm 1.44)$ months in Grade 2, and (15.35 \pm 1.52$)$ months in Grade 3, respectively (Table 1$)$.

\subsection{Blood and Lymphatic Vessels Are Increased in Recurrent Pterygia}

Because $\mathrm{CD}_{31}$ is positively expressed in both blood and lymphatic endothelial cells, while LYVE-1 is restricted to lymphatic endothelial cells [15] [16], blood vessels and lymphangions can be distinguished in tissue sections by staining for both $\mathrm{CD}_{31}$ and LYVE-1. Compared to blood vessels, lymphangions had a larger lumen and lacked erythrocytes. More blood vessels and lymphangions were observed in recurrent pterygium segments than in normal epibulbar conjunctival segments (Figure 1). Compared to control tissues, both the LMD/BMD and LVA/BVA ratios were significantly increased in recurrent pterygium (Table 2). This suggested that an increase in lymphatic vessels might be closely correlated with pterygium recurrence. Comparisons of the LMD/BMD and LVA/BVA ratios in different grades of recurrent pterygia revealed that the LMD was 0.22 times greater than the BMD and that the LVA was 0.09 times greater than the BVA in Grade 1 pterygia. In Grade 2 pterygia, the LMD was 0.3 times greater than the BMD, and the LVA was 0.1 times greater than the BVA. Finally, in Grade 3 pterygia, the LMD was 0.34 times greater than the BMD, and the LVA was 0.11 times greater than the BVA (Table 3). The increase in the LMD and LVA was significantly greater than that in the BMD and BVA, indicating that growth of the conjunctival lymphangion was not proportional to the development of blood vessels. In severe recurrent pterygia (Grade 3), lymphatic vessels grew more rapidly than blood vessels.

\subsection{Relationships between Blood Vessels and RT and between Lymphatic Vessels and RT}

To elucidate the correlation between blood vessels and pterygium recurrence, we examined the BMD, BVA, and RT values in Grade 1, 2, and 3 recurrent pterygia. Additionally, the associations between BMD and RT and between BVA and RT were analyzed. Our data indicated an important correlation between BVA and RT in pterygia of all grades, while BMD was correlated with RT only in Grade 1 pterygia. Moreover, no significant correlation was found between BMD and RT in Grade 2 and Grade 3 pterygia. However, in all three grades of pterygia, both the LMD and LVA were closely related to RT (Figure 2). This finding suggested a potential closer relationship between lymphangiogenesis and recurrent pterygia formation than between hemangiogenesis and recurrent pterygia. 
Table 1. Dimension of normal controls vs recurrent pterygia.

\begin{tabular}{ccccc}
\hline & \multirow{2}{*}{ Normal controls } & \multicolumn{3}{c}{ Recurrent pteryia } \\
\cline { 3 - 5 } & & Grade 1 & Grade 2 & Grade 3 \\
\hline Number & 7 & 14 & 20 & 14 \\
Age (years) & $60.8 \pm 11.8$ & $58.8 \pm 16.6$ & $60.5 \pm 17.8$ & $52.9 \pm 21.5$ \\
Female/male & $4 / 3$ & $7 / 7$ & $12 / 8$ & $9 / 5$ \\
RT (months) & & $25.14 \pm 4.49$ & $19.92 \pm 1.44$ & $15.35 \pm 1.52$ \\
\hline
\end{tabular}

Table 2. Comparison of lymphangiogenesis/hemangiogenesis ratios in recurrent pterygia vs controls.

\begin{tabular}{cccc}
\hline Patients & Number & LMD/BMD (\%) & LVA/BVA (\%) \\
\hline Controls & 7 & $13.99 \pm 3.15$ & $7.10 \pm 0.23$ \\
Recurrent pterygia & 48 & $28.92 \pm 2.65$ & $10.14 \pm 0.43$ \\
P values & & 0.0001 & 0.001 \\
\hline
\end{tabular}

Compared with controls, the ratios of LMD/BMD and LVA/BVA were significantly increased.

Table 3. Comparison evaluation of lymphangiogenesis vs hemangiogenesis in recurrent pterygia.

\begin{tabular}{cccc}
\hline Recurrent pterygia & Number & LMD/BMD (\%) & LVA/BVA (\%) \\
\hline Grade 1 Pterygium & 14 & $22.10 \pm 2.80$ & $8.94 \pm 0.18$ \\
Grade 2 Pterygium & 20 & $29.60 \pm 1.94^{*}$ & $10.22 \pm 0.65^{\star}$ \\
Grade 3 Pterygium & 14 & $33.87 \pm 0.93^{*+}$ & $11.22 \pm 0.63^{*+}$ \\
\hline
\end{tabular}

Compared with Grade $1,{ }^{*} \mathrm{P}<0.05$, Compared with Grade $2,{ }^{+} \mathrm{P}<0.05$.

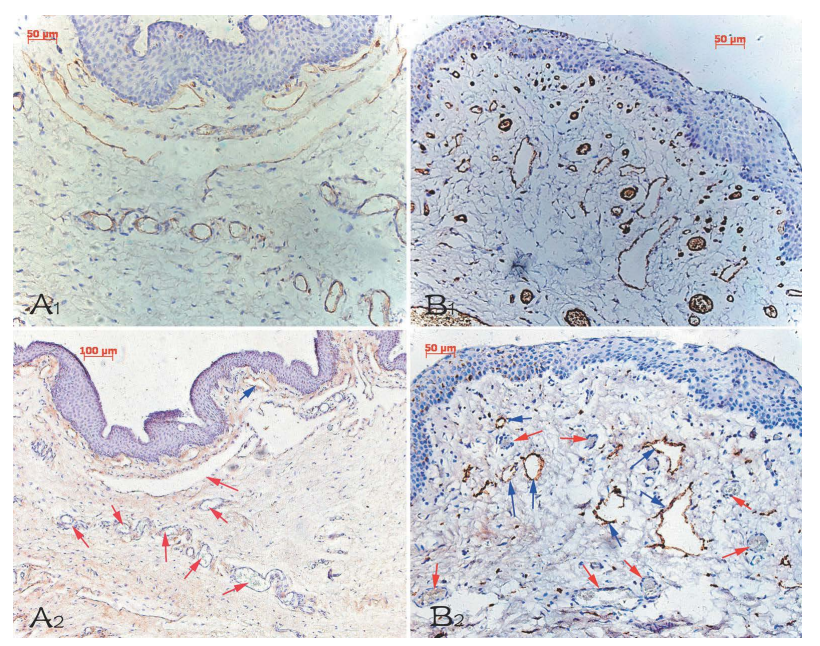

Figure 1. Cluster of differentiation 31 (CD31) and lymphatic vessel endothelial hyaluronan receptor 1 (LYVE-1) immunohistochemistry for human normal conjunctiva and recurrent petrygia. There was a certain number of $\mathrm{CD}_{31}{ }^{(+)}$LYVE- $1^{(-)}$blood vessels but only a few $\mathrm{CD}_{31}{ }^{(+)}$LYVE- $1^{(+)}$lymphatic vessels in normal epibulbar conjunctivae segments. Both blood and lymphatic vessels were increased in recurrent pterygium, especially for lymphatic vessels. There were quite a few lymphatic vessels, which were positive for CD31 and LYVE-1, having a larger lumen without erythrocytes, and locating in the stromal layer of pterygium (A: controls; B: recurrent pterygia. Footnote $1: \mathrm{CD}_{31}$ immunohistochemistry; Footnote 2: LYVE-1 immunohistochemistry; Red arrows: blood vessels; Blue arrows: lymphatic vessels. Magnification for immunohistochemistry $\times 200$ ). 

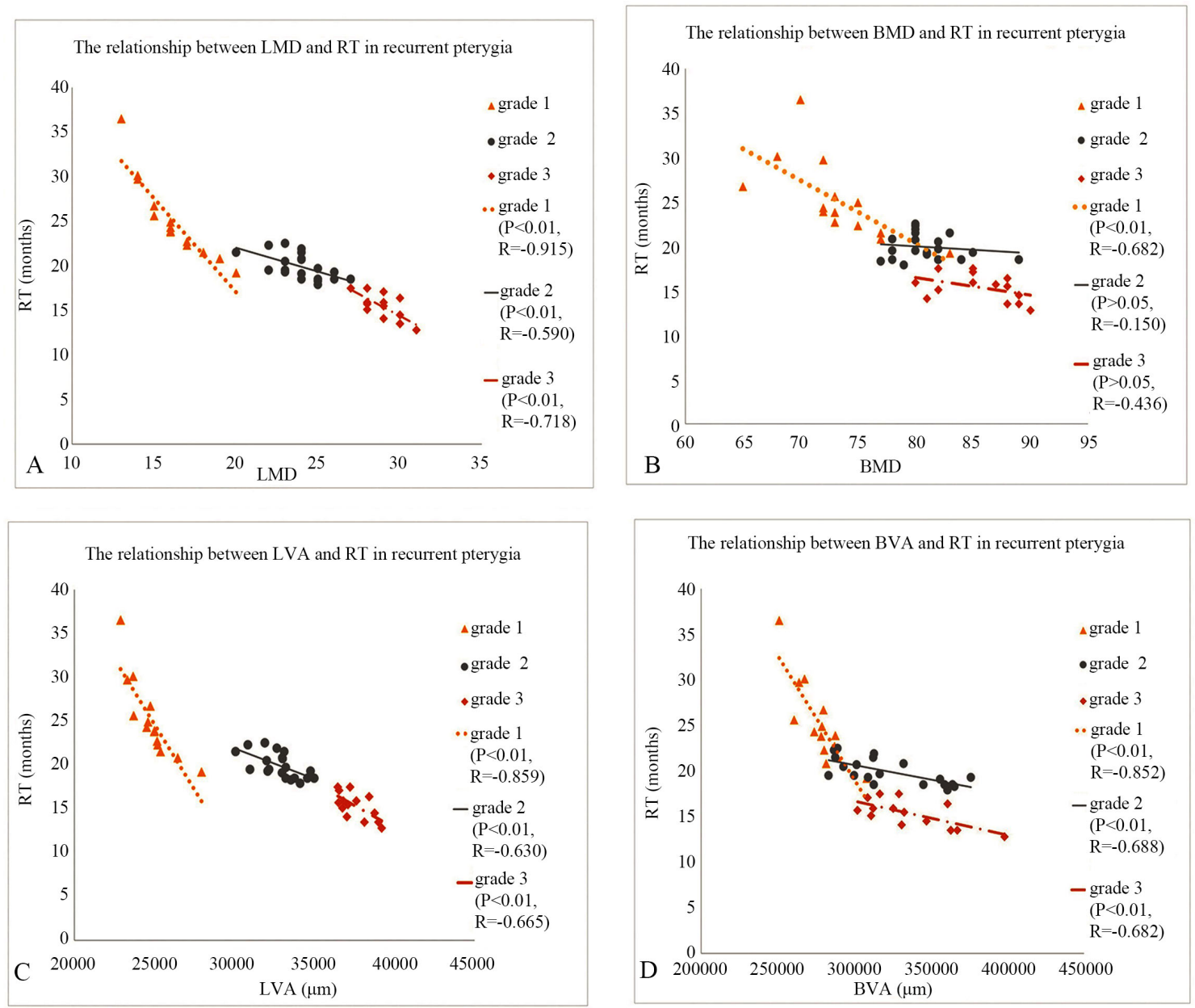

Figure 2. The relationships between LMD, LVA, BMD, BVA and RT in recurrent pterygia. Although BMD was correlated with RT in Grade 1 pterygia $(\mathrm{P}<0.05)$, there was no significant correlation between BMD and RT in Grade 2 and Grade 3 pterygia (A) $(\mathrm{P}>0.05)$. However, in pterygia at all three grades, BVA, LMD and LVA were closely related to RT (B-D) (all P values $<0.05$ ).

\section{Discussion}

Ocular lymphatic vessels facilitate the transport of antigens, thus accelerating the presentation of antigens and playing an important role in ocular immunity. However, because ocular lymphatic vessels are colorless and transparent, they often cannot be identified under a slit lamp; thus, the study of lymphangiogenesis is lagging far behind the study of hemangiogenesis [10]. Recently, new lymphatic vessel formation was observed in both primary and recurrent pterygia, providing a basis for anti-lymphatic or/and anti-vascular therapy for pterygia [7] [8] [17]. However, compared to hemangiogenesis, lymphangiogenesis cannot be easily evaluated to determine the effects of drugs targeting lymphatic metastasis in pterygia (if available) because of the lack of visibility of conjunctival lymphatics. Currently, some studies of corneal lymphangiogenesis have shown that corneal hemangiogenesis occurs in parallel to lymphangiogenesis during the course of keratitis, transplant rejection, trauma, limbal insufficiency, and suture-induced corneal neovascularization [18]-[24]. If such a parallel exists between arteries 
and lymphangions in pterygia, the number of lymphangions can be indirectly assessed by examining the number of arteries, which are readily identifiable under a slit lamp.

However, changes in the number of lymphangions, as indicated by measures such as LMD, do not fully represent changes in lymphangiogenesis. The same is true of hemangiogenesis, which is not fully represented by BMD. In addition to LMD and BMD, the LVA and BVA are important indicators for the measurement of lymphatic vessels and blood vessels, respectively. In previous studies, both LMD and LVA were relevant to the width and region of pterygia, and LVA was also correlated with the extension of pterygia. This result suggested that LVA was more closely related to pterygium development than LMD [25]. Therefore, in this study, we used both the LMD and LVA to evaluate lymphangiogenesis. The increases in LMD and LVA were significantly greater than those of BMD and BVA, especially in Grade 2 and 3 pterygia. Together with our previous studies on the relationship between arteries and lymphangions in recurrent pterygia, the present data suggest that the relationship between new vessels and lymphangions is significant but not parallel. Therefore, we cannot simply assess the condition of conjunctival lymphangiogenesis by the state of hemangiogenesis, especially in substantial recurrent pterygia.

In fact, the parallel growth of new corneal blood vessels and lymphatic vessels is controversial to some extent. Lymphangions unaccompanied by arteries have been observed toward the center of corneas in a mouse model of dry eye disease (DED) induced by high-flow desiccated air. This finding suggests the lack of a parallel relationship between corneal hemangiogenesis and corneal lymphangiogenesis in low-level inflammation associated with DED [26] [27]. In a previous study, the progression and regression of corneal inflammation, hemangiogenesis, and lymphangiogenesis were not synchronized in alkali-burned rat corneas. Penetrating corneal transplantation was performed at the most severe and mildest stages of keratitis, hemangiogenesis and lymphangiogenesis after alkaline burns, and the rejection of corneal grafts was compared. Our study showed that corneal lymphatic vessels played a more significant role in allograft rejection than did corneal hemangiogenesis [28]. Since blood vessels and lymphatic vessels in pterygia are closely related, similar to hemangiogenesis and lymphangiogenesis in the inflammatory cornea, the question of which factor plays a more significant role in the recurrence of pterygium is worth discussing.

In an early study [29], we examined hemangiogenesis and lymphangiogenesis in recurrent pterygia and found a significant increase in LMD and BMD, indicating that both blood and lymphatic vessels played a role in pterygia recurrence. The data also showed that LMD was dramatically increased in Grade 3 pterygia and suggested that lymphatic vessels might play a more important role than blood vessels in serious recurrent pterygia. However, the study could not show the relationship between LMD or BMD and RT, which indicated the need to compare the roles of lymphatic vessels and blood vessels in pterygium recurrence. In addition, two important indicators, LVA and BVA, were lacking in the 
study, which decreased the accuracy of measurement of lymphatic vessels and blood vessels. In the current study, we compared differences in two indicators, the LMD/BMD and LVA/BVA ratios, between the normal conjunctiva and recurrent pterygia. Unlike other indicators, such as LMD, LVA, BMD, and BVA, which only focus on the outgrowth of lymphatic vessels or blood vessels alone, the LMD/BMD and LVA/BVA ratios are correlated with the changes of both, which is more conducive to comparing the proportion of lymphangiogenesis and hemangiogenesis. Our data showed that both LMD/BMD and LVA/BVA were significantly increased in recurrent pterygia. This result suggested that lymphangions were more closely associated with the recurrence of pterygia. Subsequently, we divided patients with pterygium into three grades according to the scores assigned in a slit lamp examination (Grade 1 to 3 ) and focused on the changes in LMD/BMD and LVA/BVA in different grades of recurrent pterygia. Our data showed that a higher pterygium grade corresponded to higher LMD/BMD and LVA/BVA ratios, indicating that lymphatic vessels play a more important role than blood vessels not only in the recurrence of pterygium, but also in its proliferation process.

In an earlier study, we observed an important negative correlation between RT and LMD rather than BMD [30]. Unlike our earlier study, which was a prospective study that involved tracking patients with primary pterygium from initial pterygium surgery to the time of recurrence, the current study was a retrospective study, and RT was determined according to the description of patients with recurrent pterygium. Our earlier study focused on hemangiogenesis and lymphangiogenesis in primary pterygia, whereas changes in arteries and lymphangions in histological sections of recurrent pterygia were examined in the current study. Moreover, the present study included twice the number of patients with recurrent pterygia compared to the previous study, and important indexes such as LVA and BVA were added to evaluate the area of blood and lymphatic vessels to obtain more accurate results. We observed an important negative correlation between LMD and RT but not a close correlation between BMD and RT, similar to the conclusions of earlier studies. However, similar to LVA, an important negative correlation was observed between BVA and RT. The results suggested that both arteries and lymphangions were important factors in pterygium recurrence, but pterygium recurrence was more closely related to lymphatic vessels.

However, there are some limitations in the study. First, although we have elucidated a close relationship between lymphangiogenesis and RT, there is still no clear evidence how lymphatic vessels lead to the recurrence of pterygium. Whether lymphangiogensis itself or an immunological process associated with lymphangiogenesis indirectly contributes to the pterygium proliferation and/or invasion (or conjunctival tissue proliferation) in vitro requires further research. Second, the number of the participants in this study was slightly insufficient. Further studies of larger samples are warranted.

In summary, our study revealed that conjunctival lymphangiogenesis might play a more important role than hemangiogenesis in the recurrence of pterygia. 
Anti-lymphangiogenic therapeutic strategies should be investigated to reduce the growth and recurrence of pterygia.

\section{Acknowledgements}

Foundation: The study was supported by the Guangdong Natural Science Foundation (No. 2015B020226003 and No. 2016A030313208).

\section{Conflicts of Interest}

The authors declare no conflicts of interest regarding the publication of this paper.

\section{References}

[1] Mak, R.K., Chan, T.C., Marcet, M.M., Choy, B.N., et al. (2017) Use of Anti-Vascular Endothelial Growth Factor in the Management of Pterygium. Acta Ophthalmologica, 95, 20-27. https://doi.org/10.1111/aos.13178

[2] Yalcin Tok, O., Burcu Nurozler, A., Ergun, G., Akbas Kocaoglu, F. and Duman, S. (2008) Topical Cyclosporine a in the Prevention of Pterygium Recurrence. Ophthalmologica, 222, 391-396. https://doi.org/10.1159/000151740

[3] Ibáñez, M., Eugarrios, M.F. and Calderón, D.I. (2009) Topical Cyclosporin A and Mitomycin C Injection as Adjunctive Therapy for Prevention of Primary Pterygium Recurrence. Ophthalmic Surgery, Lasers \& Imaging, 40, 239-244. https://doi.org/10.3928/15428877-20090430-03

[4] Awdeh, R.M., DeStafeno, J.J., Blackmon, D.M., Cummings, T.J. and Kim, T. (2008) The Presence of T-Lymphocyte Subpopulations (CD4 and CD8) in Pterygia: Evaluation of the Inflammatory Response. Advances in Therapy, 25, 479-487. https://doi.org/10.1007/s12325-008-0056-4

[5] Tekelioglu, Y., Turk, A., Avunduk, A.M. and Yulug, E. (2006) Flow Cytometrical Analysis of Adhesion Molecules, T-Lymphocyte Subpopulations and Inflammatory Markers in Pterygium. Ophthalmologica, 220, 372-378. https://doi.org/10.1159/000095863

[6] Rohrbach, I.M., Starc, S. and Knorr, M. (1995) Predicting Recurrent Pterygium Based on Morphologic and Immunohistologic Parameters. Ophthalmologe, 92, 463-468.

[7] Zhao, W., Wang, T., Deng, J., Zhong, L., Huang, W. and Ling, S. (2016) Conjunctival Lymphangiogenesis Was Associated with the Degree of Aggression in Substantial Recurrent Pterygia. Journal of Ophthalmology, 2016, Article ID: 1592514. https://doi.org/10.1155/2016/1592514

[8] Ling, S., Li, Q., Lin, H., Li, W., Wang, T., Ye, H., Yang, J., Jia, X. and Sun, Y. (2012) Comparative Evaluation of Lymphatic Vessels in Primary versus Recurrent Pterygium. Eye (London), 26, 1451-1458. https://doi.org/10.1038/eye.2012.194

[9] Zhong, W., Montana, M., Santosa, S.M., Isjwara, I.D., Huang, Y.H., et al. (2018) Angiogenesis and Lymphangiogenesis in Corneal Transplantation-A Review. Survey of Ophthalmology, 63, 453-479. https://doi.org/10.1016/j.survophthal.2017.12.008

[10] Cursiefen, C., Chen, L., Dana, M.R. and Streilein, J.W. (2003) Corneal Lymphangiogenesis: Evidence, Mechanisms, and Implications for Corneal Transplant Immunology. Cornea, 22, 273-281. https://doi.org/10.1097/00003226-200304000-00021

[11] Regenfuss, B., Bock, F. and Cursiefen, C. (2012) Corneal Angiogenesis and Lym- 
phangiogenesis. Current Opinion in Allergy and Clinical Immunology, 12, 548-554. https://doi.org/10.1097/ACI.0b013e328357b4a2

[12] Zhu, Y., Li, L., Reinach, P.S., Li, Y., Ge, C., Qu, J. and Chen, W. (2018) Corneal Collagen Cross-Linking with Riboflavin and UVA Regulates Hemangiogenesis and Lymphangiogenesis in Rats. Investigative Ophthalmology \& Visual Science, 59, 3702-3712. https://doi.org/10.1167/iovs.17-23036

[13] Hayashi, T., Usui, T. and Yamagami, S. (2016) Suppression of Allograft Rejection with Soluble VEGF Receptor 2 Chimeric Protein in a Mouse Model of Corneal Transplantation. The Tohoku Journal of Experimental Medicine, 239, 81-88. https://doi.org/10.1620/tjem.239.81

[14] Ferrari, G., Bignami, F. and Rama, P. (2015) Tumor Necrosis Factor-Alpha Inhibitors as a Treatment of Corneal Hemangiogenesis and Lymphangiogenesis. Eye Contact Lens, 41, 72-76. https://doi.org/10.1097/ICL.0000000000000071

[15] Mitrofanova, I., Zavyalova, M., Riabov, V., Cherdyntseva, N. and Kzhyshkowska, J. (2018) The Effect of Neoadjuvant Chemotherapy on the Correlation of $\mathrm{Tu}$ mor-Associated Macrophages with CD31 and LYVE-1. Immunobiology, 223, 449-459. https://doi.org/10.1016/j.imbio.2017.10.050

[16] Bock, F., Onderka, J., Braun, G., Schneider, A.C., Hos, D., Bi, Y., Bachmann, B.O. and Cursiefen, C. (2016) Identification of Novel Endogenous Anti(Lymph)angiogenic Factors in the Aqueous Humor. Investigative Ophthalmology \& Visual Science, 57, 6554-6560. https://doi.org/10.1167/iovs.15-18526

[17] Cimpean, A.M., Poenaru Sava, M., Raica, M. and Ribatti, D. (2011) Preliminary Evidence of the Presence of Lymphatic Vessels Immunoreactive for D2-40 and Prox-1 in Human Pterygium. Oncology Reports, 26, 1111-1113.

[18] Cursiefen, C., Maruyama, K., Jackson, D.G., Streilein, J.W. and Kruse, F.E. (2006) Time Course of Angiogenesis and Lymphangiogenesis after Brief Corneal Inflammation. Cornea, 25, 443-447. https://doi.org/10.1097/01.ico.0000183485.85636.ff

[19] Park, P.J., Chang, M., Garg, N., Zhu, J., Chang, J.H. and Shukla, D. (2015) Corneal Lymphangiogenesis in Herpetic Stromal Keratitis. Survey of Ophthalmology, 60, 60-71. https://doi.org/10.1016/j.survophthal.2014.06.001

[20] Zheng, Y., Lin, H. and Ling, S. (2011) Clinicopathological Correlation Analysis of (Lymph) Angiogenesis and Corneal Graft Rejection. Molecular Vision, 17, 1694-1700.

[21] Ling, S.Q., Liu, C., Li, W.H., Xu, J.G. and Kuang, W.H. (2010) Corneal Lymphangiogenesis Correlates Closely with Hemangiogenesis after Keratoplasty. International Journal of Ophthalmology, 3, 76-79.

[22] Ling, S., Lin, H., Xiang, D., Feng, G. and Zhang, X. (2008) Clinical and Experimental Research of Corneal Lymphangiogenesis after Keratoplasty. Ophthalmologica, 222, 308-316. https://doi.org/10.1159/000144030

[23] Chang, J.H., Putra, I., Huang, Y.H., Chang, M., Han, K., Zhong, W., Gao, X., Wang, S., Dugas-Ford, J., Nguyen, T., Hong, Y.K. and Azar, D.T. (2016) Limited versus Total Epithelial Debridement Ocular Surface Injury: Live Fluorescence Imaging of Hemangiogenesis and Lymphangiogenesis in Prox1-GFP/Flk1:Myr-mCherry Mice. Biochimica et Biophysica Acta, 1860, 2148-2156. https://doi.org/10.1016/j.bbagen.2016.05.027

[24] Giacomini, C., Ferrari, G., Bignami, F. and Rama, P. (2014) Alkali Burn versus Suture-Induced Corneal Neovascularization in C57BL/6 Mice: An Overview of Two Common Animal Models of Corneal Neovascularization. Experimental Eye Research, 121, 1-4. https://doi.org/10.1016/j.exer.2014.02.005

[25] Liu, L., Ling, S.Q., Li, Q.L., Wang, T., Ye, H., Yang, J.Z. and Jia, X.H. (2012) Rela- 
tions between Lymphangiogenesis and the Size of Pterygium. International Journal of Ophthalmology, 5, 312-316.

[26] Goyal, S., Chauhan, S.K., El Annan, J., Nallasamy, N., Zhang, Q. and Dana, R. (2010) Evidence of Corneal Lymphangiogenesis in Dry Eye Disease: A Potential Link to Adaptive Immunity? Archives of Ophthalmology, 128, 819-824.

https://doi.org/10.1001/archophthalmol.2010.124

[27] Goyal, S., Chauhan, S.K. and Dana, R. (2012) Blockade of Prolymphangiogenic Vascular Endothelial Growth Factor C in Dry Eye Disease. Archives of Ophthalmology, 130, 84-89. https://doi.org/10.1001/archophthalmol.2011.266

[28] Ling, S., Qi, C., Li, W., Xu, J. and Kuang, W. (2009) Crucial Role of Corneal Lymphangiogenesis for Allograft Rejection in Alkali-Burned Cornea Bed. Clinical and Experimental Ophthalmology, 37, 874-883. https://doi.org/10.1111/j.1442-9071.2009.02178.x

[29] Qi, C.X., Zhang, X.D., Yuan, J., Yang, J.Z., Sun, Y., Wang, T., Ye, H. and Ling, S.Q. (2012) Relationship between Angiogenesis and Lymphangiogenesis in Recurrent Pterygium. International Journal of Ophthalmology, 5, 655-660.

[30] Lin, H., Luo, L., Ling, S., Chen, W., Liu, Z., Zhong, X., Wu, C., Chen, W. and Liu, Y. (2013) Lymphatic Microvessel Density as a Predictive Marker for the Recurrence Time of Pterygium: A Three-Year Follow-Up Study. Molecular Vision, 19, 166-173. 